\title{
Konsenserfahrung von International Uveitis Study Group, Intraocular Inflammation Society und Foster Ocular Inflammation Society zur Uveitis in der Zeit der COVID-19-Infektion* \\ Evolving Consensus of International Uveitis Study Group, Intraocular Inflammation Society, and Foster Ocular Inflammation Society with Uveitis in the Time of COVID-19 Infection
}

\footnotetext{
Autoren Rupesh Agarwal ${ }^{7}$

Institute

1 Universitäts-Augenklinik, Universitätsklinikum Tübingen

2 Retina and Inflammation Unit, Microinvasive Ocular Surgery Clinic, Lausanne, Schweiz

3 Ophthalmology, Advance Eye Centre, Post Graduate Institute of Medical Education and Research, Chandigarh, Indien

4 Ophthalmology, Moorfields Eye Hospital NHS Foundation Trust, London, Vereinigtes Königreich von Großbritannien und Nordirland

5 Byers Eye Institute, Spencer Center for Vision Research, Stanford, USA

6 Ophthalmology, Singapore National Eye Center, Singapur

7 Ophthalmology, Singapore Eye Research Institute, Singapur
}

Manfred Zierhut ${ }^{1}$, Marc D. de Smet ${ }^{2}$, Vishali Gupta ${ }^{3}$, Carlos Pavesio ${ }^{4}$, Quan Dong Nguyen ${ }^{5}$, Soon-Phaik Chee ${ }^{6}$,

Schlüsselwörter

Uveitis, Konsensus, COVID-19, Immunsuppression

Key words

uveitis, consensus, COVID-19, immune suppression

$\begin{array}{ll}\text { eingereicht } & 5.6 .2020 \\ \text { angenommen } & 15.6 .2020 \\ \text { online publiziert } & 14.8 .2020\end{array}$

\section{Einleitung}

Dieses Dokument fasst die Erfahrungen der International Uveitis Study Group (IUSG), der Intraocular Inflammation Society (IOIS) und der Foster Ocular Inflammation Society (FOIS) zusammen und kann als Leitfaden für die Behandlung von Uveitispatienten im Zeitalter der COVID-19-Pandemie dienen. Dieser Konsens ist
Bibliografie

Klin Monatsbl Augenheilkd 2020; 237: 1124-1128

DOI 10.1055/a-1201-3277

ISSN 0023-2165

(C) 2020. Thieme. All rights reserved.

Georg Thieme Verlag KG, Rüdigerstraße 14,

70469 Stuttgart, Germany

Korrespondenzadresse

Prof. Manfred Zierhut

Universitäts-Augenklinik, Universitätsklinikum Tübingen

Elfriede-Aulhorn-Straße 7, 72076 Tübingen

Tel.: + 49(0) 70712984008 , Fax: + 49(0) 7071294762

manfred.zierhut@med.uni-tuebingen.de

\section{ZUSAMMENFASSUNG}

Dieses Dokument fasst die Erfahrungen der International Uveitis Study Group (IUSG), der Intraocular Inflammation Society (IOIS) und der Foster Ocular Inflammation Society (FOIS) zusammen und kann als Leitfaden für die Behandlung von Uveitispatienten im Zeitalter der COVID-19-Pandemie dienen.

\section{ABSTRACT}

This document summarizes the experience of the International Uveitis Study Group (IUSG), the Intraocular Inflammation Society (IOIS) and the Foster Ocular Inflammation Society (FOIS) and can aid as a guide for the treatment of uveitis patients in the era of COVID-19 pandemic. auch auf der Website der IUSG (https://www.iusg.net/uploads/ images/IUSG\%20Library/001-Consensus-Experience-Document.

* Dieses Statement erscheint auch in der Zeitschrift Ocular Immunology and Inflammation, Taylor \& Francis, London. 
pdf), der IOIS und der FOIS veröffentlicht. Er ist daher jedem Arzt zugänglich, der Rat für seine Uveitispatienten sucht.

\section{Risikofaktoren}

Dies sind die derzeit bekannten Risikofaktoren für eine schwere COVID-19-Infektion:

1. Alter über 60 Jahre (in einigen Ländern liegt die Grenze bei 65 Jahren), mit einem signifikanten Risiko von 70 Jahren und darüber

2. Komorbidität: Herz-Kreislauf-Erkrankungen > Erkrankungen der Atemwege > arterielle Hypertonie (HTN), Diabetes mellitus (DM), Fettleibigkeit (BMI > 40) [1]

3. Kontakt mit infizierten Personen (insbesondere in der Familie) oder kürzliche Reisen in stark betroffene Länder oder Regionen

4. Beschäftigte im Gesundheitswesen (erhöhte Viruslast/Exposition aufgrund fehlender geeigneter persönlicher Schutzausrüstung [PSA])

5. Verwendung von mittel- bis hochdosierten Kortikosteroiden $[1,2]$

\section{Uveitissituationen}

Im Allgemeinen gibt es 3 mögliche Uveitisszenarien:

Bei stabilen Uveitispatienten unter systemischer immunmodulatorischer Therapie (IMT)

1. ohne klinische Anzeichen einer COVID-19-Infektion (präsymptomatisch oder asymptomatisch)

2. entweder mit bestätigter COVID-19- oder mit klinischen Anzeichen einer COVID-19-Infektion

3. ein neuer Uveitispatient, der eine Immunsuppression benötigt, oder ein Uveitispatient mit einer Exazerbation, die eine erhöhte IMT-Dosis erfordert

\section{Szenario 1: ein Uveitispatient ohne Bestätigung der klinischen Anzeichen einer COVID-19-Infektion}

Diesen Patienten wurde meist bereits zuvor erläutert, wie sie eine Infektion vermeiden können, als die IMT begonnen worden war. Dadurch kann man erwarten, dass sie bereits den Wert des Händewaschens zur Vermeidung von Infektionen kennen, persönliche Hygiene und Vermeiden von Menschenmassen praktizieren.

Der Augenarzt sollte diese Patienten darin bestärken, persönliche Schutzvorrichtungen zu verwenden, und sie ermutigen, alle auf lokaler oder nationaler Ebene vorgeschlagenen Präventionsmaßnahmen zu befolgen. Am effektivsten sind angemessene soziale Distanzierung, das Tragen von Masken (Richtlinien unterscheiden sich je nach Land) und häufiges Händewaschen bei Kontakt mit Oberflächen.

In bestimmten europäischen Ländern wurden nach Beginn der Pandemie Risikogruppen entweder persönlich telefonisch oder per Post/E-Mail kontaktiert, um personalisierte, auf die Art der Uveitis bzw. der IMT abgestimmte Empfehlungen zu erläutern. Dieser Kontakt trug bei unseren Patienten bisher dazu bei, dass sie auf die zu treffenden Maßnahmen aufmerksam gemacht werden konnten, um eine Infektion mit COVID-19 zu vermeiden. Diese Maßnahmen umfassen:

- so viel wie möglich zu Hause bleiben,
- soziale Distanzierung üben (Abstand von 1,5 bis $2 \mathrm{~m}$ zu anderen Personen), sei es auf der Straße, in Geschäften oder in Wartezimmern,

- Tragen einer Maske, wenn sie sich in der Nähe von Nichthaushaltsmitgliedern oder an überfüllten Orten befinden,

- Hände häufig mindestens 20 s lang mit Seife zu waschen, insbesondere nach dem Berühren von Oberflächen (Transaktionsautomaten, Türknöpfen, Aufzugsknöpfen und Lichtschaltern); der direkte Kontakt von kontaminierten Oberflächen ist wohl eine der wichtigen Übertragungsarten [3]

- das Gesicht nicht zu berühren, wenn sie ihre Hände nicht waschen konnten

- das Tragen von Handschuhen; auch Leder- oder Winterhandschuhe können eine Option sein, wenn keine Latex-/Vinylhandschuhe verfügbar sind.

Wenn sich der Patient unwohl fühlt, sollte er sich an seine Arztpraxis wenden, die ihn über die Dringlichkeit eines Termins beraten sollte. Dies kann je nach Land leicht möglich oder schlecht möglich sein. In einigen Ländern dürfen Praxen COVID-Verdächtige nur dann empfangen, wenn sie mit Isolationsräumen für verdächtige Patienten ausgestattet sind und geeignete Barriere-PSA verfügbar sind (Schürzen, N95- oder gleichwertige Masken sowie Gesichtsschutz oder Brille). Alternativ können solche Patienten an ein geeignetes Diagnosezentrum überwiesen werden. Insbesondere im Frühjahr und Herbst sollte man daran denken, dass andere Viruserkrankungen vorhanden sein könnten. Ein entsprechender COVID-19-Nachweis ist daher zwingend erforderlich, wenn die entsprechenden Symptome vorliegen. Abhängig von den örtlichen Verhältnissen sollten Patienten unter Immunsuppression als „Risikogruppe“ eingestuft werden und eine frühzeitige COVID-19-Testung erhalten.

Im klinischen Bereich sollten Risikopatienten vorzugsweise früher am Tag und zu einem anderen Zeitpunkt als Patienten außerhalb von Risikogruppen gesehen werden. Es sollte eine Höchstanzahl von Patienten im Wartezimmer festgelegt werden, die sozialen Abstände ermöglicht werden und ein alkoholisches Desinfektionsmittel bereitgestellt sein. Begleitpersonen sollten, falls dies möglich ist, außerhalb des Hauses warten. Jeder sollte eine Maske tragen. Warteraum und die Untersuchungsräume sollten ausreichend gelüftet sein. Türen sollten offen bleiben, einschließlich des Eingangs zum Büro, um die Luftzirkulation zu erhöhen. Spaltlampen sollten mit Abschirmungen ausgestattet sein, und der Augenarzt sollte einen sicheren Abstand zu den Patienten halten, wenn er die Situation bespricht. Konsultationen sollten auf ein Minimum beschränkt bleiben (5 min, wenn möglich). Wir empfehlen, die erfolgten Untersuchungen (Scans, Gesichtsfelder, Laborwerte) zu lesen, bevor der Patient erneut hereingebeten wird. Die Aktendurchsicht von Patienten 1 oder 2 Tage vor ihrem geplanten Besuch auf mögliche Risikofaktoren kann eventuell verhindern, solche Patienten in der Sprechstunde zu sehen. Wir empfehlen virtuelle Konsultationen (oder auch nur Telefonkontakt) für Patienten mit geringem Rezidivrisiko und bei stabilem Befund.

Die erste Verteidigungslinie gegen eine Infektion ist die angeborene Immunität. Wenn also die Gesamtzahl der weißen Blutkörperchen des Patienten über der Untergrenze des Normalwerts (4000 pro $\mu$ l) liegt, verringert sich das Infektionsrisiko (siehe: 
https://www.ncbi.nlm.nih.gov/books/NBK261/). Immunmodulatorische Mittel, die wie Ciclosporin-A durch Reduktion von T-Zellen wirken, sind im Allgemeinen in moderaten Dosen sicher und scheinen das Risiko für Virusinfektionen wahrscheinlich nicht zu erhöhen, allerdings mit der Ausnahme von Infektionen mit dem Varizella-Zoster-Virus (VZV; siehe: https://www.ncbi.nlm.nih.gov/ books/NBK47401/). Die Laborkontrollen sollten beibehalten und vorzugsweise in der Nähe der Wohnung durchgeführt werden, um Expositionen weitestgehend zu minimieren.

Das Virus bindet an seine Zielzellen über Renin-AngiotensinRezeptoren (ACE-2), die an den Epithelzellen der Lunge, des Darms, der Niere, der Blutgefäße und sogar der Bindehaut exprimiert werden. Die Expression von ACE-2 ist bei Patienten mit Typ1- und Typ-2-DM oder Patienten, die mit ACE-Inhibitoren und ACE-2-Rezeptorblockern (ARBs) behandelt werden, signifikant erhöht. ACE-2-Hemmer reduzieren Entzündungen und wurden kürzlich gegen entzündliche Lungenerkrankungen, Karzinome, Diabetes und Bluthochdruck vorgeschlagen. ACE-Hemmer verursachen eine Hochregulation von ACE-2 und könnten für eine Infektion mit COVID-19 prädisponieren. Aufgrund von ACE-2-Polymorphismen scheint hierbei eine genetische Veranlagung für ein erhöhtes Risiko für eine SARS-CoV-2-Infektion zu bestehen, die mit DM, HTN und Schlaganfall verbunden ist, und dies insbesondere in asiatischen Populationen. Die Rolle von ACE-Hemmern in Bezug auf die Virulenz der COVID-19-Infektion ist jedoch nicht bekannt. Kardiologen empfehlen derzeit nicht, ACE-Hemmer bei diesen Patienten zu stoppen, da dies mehr Schaden als Nutzen verursachen würde $[4,5]$. Es sollte aber klar sein, dass Uveitispatienten mit Komorbiditäten wie DM, HTN und Herzerkrankungen ein höheres Risiko haben, an COVID-19 zu erkranken [1].

Auch wenn Patienten gut darüber informiert sind, wie sie sich vor Infektionen schützen können, empfehlen wir Ihnen oder Ihren Mitarbeitern trotzdem, sich telefonisch an Ihre Patienten zu wenden, die IMTs erhalten. Dies können Ratschläge zum Verhalten sein oder einige Richtlinien, was zu tun ist, wenn sie COVID-19positiv werden. Die bisherige Erfahrung zeigt, dass viele Patienten beruhigt sind und an die Bedeutung von Distanzierungsmaßnahmen erinnert werden. Einige Patienten tendieren von sich aus dazu, ohne ärztlichen Rat ihre Medikamente (besonders IMT) abzusetzen. Eine solche Kommunikation ist natürlich auch bei der Betreuung von Kindern mit IMT von großer Bedeutung und bietet Augenärzten die Möglichkeit, den Stand der Behandlung (Reduktion der Therapie?) und alternative Überbrückungswege zu erörtern. Falls möglich, sollte dabei durchaus die Therapieplanung über die nächsten Monate besprochen werden, da die aktuellen Prognosen zur Pandemie von einem Verlauf von bis zu 18 Monaten ausgehen [7].

Daher sind wir uns darin einig, dass bei COVID-19-negativen Patienten die IMT beibehalten werden sollten. Die Notwendigkeit einer fortgesetzten Therapie sollte aber neu bewertet und die Steroiddosis minimiert werden, wann immer dies möglich ist.

\section{Szenario 2: Patient mit bestätigter COVID-19- Infektion oder mit klinischen Anzeichen einer COVID-19-Infektion}

Falls der Patient klinische Symptome entwickelt, sollte eine Bestätigung der Diagnose baldigst erfolgen, da andere virale Ursachen vorliegen könnten, die COVID-19 imitieren können. Bitte erinnern Sie Ihren Patienten daran, den Arzt über alle Medikamente zu informieren, die er gegen seine Uveitis einnimmt.

Wenn der Patient asymptomatisch ist, empfehlen wir, die IMT zusammen mit der Blutüberwachung fortzusetzen und die Dosis zu reduzieren, wenn der Wert der Leukozyten unter 4000/ $\mu$ l fällt.

Symptomatische Patienten sollten ihre konventionelle IMT und biologische Therapie vorübergehend abbrechen (außer Interferon und Tocilizumab, siehe später). Patienten, die TNF-Blocker (TNF: Tumor-Nekrose-Faktor) einnehmen, sollten ihre nächsten geplanten subkutanen Dosen auslassen, bis es ihnen besser geht.

Wenn möglich, sollte auf lokale (intravitreale Injektionen oder Medikamententräger) als Behandlungsoption übergegangen werden. Systemische Kortikosteroide müssen möglicherweise langsam reduziert werden. Dieser Schritt sollte jedoch mit dem COVID-Behandlungsteam besprochen werden. Niedrige Erhaltungsdosen < $10 \mathrm{mg} / \mathrm{Tag}$ Prednisolonäquivalent stellen möglicherweise kein signifikantes Risiko dar und sollten beibehalten werden. Wenn die Dosen über $20 \mathrm{mg}$ liegen, sollten diese (falls notwendig auch unter Verwendung einer intravitrealen Therapie) reduziert werden. Hochdosierte Steroide können zu hoher Viruslast führen, was anschließend zu einer Dysregulation des Immunsystems führen könnte [3].

In den meisten Fällen ist der Krankheitsverlauf nur von kurzer Dauer. Die Symptome sind 10-14 Tage lang nachweisbar. Tritt eine Atemstörung auf, wird dies normalerweise am 7. oder 8. Tag deutlich und es folgt eine rasche Erholung oder es zeigt sich die Notwendigkeit einer stärkeren Sauerstoffunterstützung. Sobald die Atemstörung abgeklungen ist, kann die ursprüngliche IMT wieder aufgenommen werden.

\section{Wirkung von Immunsuppressiva auf das Virus}

- Eine Immunsuppression scheint das Risiko einer COVID-19-Erkrankung nicht zu erhöhen. Eine Überprüfung der Mortalitätsund Morbiditätsberichte früherer Corona-Infektionen ergab keine Mortalität in Transplantationspatienten [8]. In Bergamo wurde von 522 Patienten mit chronisch entzündlichen Darmerkrankungen (IBD) keiner mit einer nach nachgewiesenen SARS-CoV-2-Infektion ins Krankenhaus eingeliefert, wobei statistisch gesehen 21 Aufnahmen zu erwarten gewesen wären [9]. Bhoori et al. veröffentlichten eine Reihe von 111 erwachsenen Langzeit-Lebertransplantations-Patienten mit minimaler Immunsuppression, unter denen 3 COVID-19-Todesfälle aufgetreten waren, und verglichen diese mit 3 symptomlosen SARS-CoV-2-Infektionen unter 40 kürzlich transplantierten und vollständig immunsupprimierten Patienten in diesem Zentrum. Die aufgezeichneten Todesfälle schienen eher mit den bekannten Risikofaktoren einer schweren COVID-19-Erkrankung (Alter, Vorhandensein mehrerer Begleiterkrankungen) als mit dem Grad der Immunsuppression in Zusammenhang zu stehen, und die Autoren meinten, dass Immuntherapie möglicherweise vor einer schweren Erkrankung geschützt habe [10].

- Interferon $\alpha$ und $\beta$ sowie Anti-IL-6-Medikamente (Tocilizumab) können das „Cytokine Storm Syndrom“ reduzieren [11,12]. Dies scheint einer der wesentlichen Faktoren zu sein, die während einer COVID-19-Infektion durch eine übermäßige Freiset- 
zung von Zytokinen (IL-1, IL-6, IL-18 und Interferon y) zum Tod führen. Es resultiert ein Multiorganversagen. Die IL-6-Blockade wird derzeit in einer prospektiven Studie bei COVID-19-Patienten in China untersucht.

\section{Szenario 3: neuer Uveitispatient benötigt eine Immunsuppression oder Patient hat eine Exazerbation, die eine verstärkte Immunsuppression erfordert}

Patienten mit schwerer akuter Uveitis (neue Uveitis oder Reaktivierung trotz IMT) werden traditionell primär mit hohen Dosen von oralen Steroiden oder auch i.v. Methylprednisolon versorgt. In dieser Situation sollte eine lokale Therapie (periokulare oder intravitreale Steroide) allein oder in Kombination mit niedrigeren Dosen systemischer Steroide in Betracht gezogen werden. Bei akutem Morbus Behçet sollte möglichst Interferon $\alpha$ oder $\beta$ in $\mathrm{Er}$ wägung gezogen werden. Bisher war jedoch der Verlauf bei Patienten, die sich unter Anti-TNF-Medikamenten mit SARS-CoV-2 infiziert hatten, mild, ohne dass über schwere Erkrankungen oder Todesfälle berichtet worden wäre. Die Rheumatologieverbände haben mehrere Leitlinien vorgeschlagen.

\section{Augenmanifestationen von SARS-CoV-2}

Augenmanifestationen wurden bisher auf der Ebene der Konjunktiva und der Retina festgestellt: Bindehautentzündung oder perilimbale Rötung [15] wurden beschrieben, wobei die meisten Fälle wohl bei florider Virusinfektion diagnostiziert wurden; nur in 1/38 Patienten war die Konjunktivitis ein präsentierendes Symptom [14]. Die Prävalenz von SARS-CoV-2-Nukleotiden war bei Bindehautproben (5\%) von COVID-positiven Personen gering [14] und die Persistenz bei Tränen ist nur von kurzer Dauer (12 d) [15]. Als Übertragungsweg spielt die Tränenflüssigkeit daher eher eine begrenzte Rolle [16]. Der Fall einer pseudomembranösen hämorrhagischen Konjunktivitis wurde als späte Manifestation beschrieben [17].

Über retinale Manifestationen wurden in Form von hyperreflektierenden Läsionen in den Ganglienzellen und den inneren plexiformen Schichten der Retina innerhalb des papillomakulären Bündels berichtet. Bei näherer Betrachtung wurden kleine CottonWool-Spots und Mikrohämorrhagien festgestellt. Diese Patienten wurden 11 bis 33 Tage nach Auftreten der Symptome untersucht [18]. Kürzlich wurde SARS-CoV-2 auch in Netzhautbiopsien verstorbener COVID-19-Patienten nachgewiesen [19].

\section{Labormarker für Coronavirusinfektionen [20]}

Einige Laborparameter werden durch IMT beeinflusst (z. B. Blutkörperchensenkungsgeschwindigkeit [BSG]) oder sind bei Verwendung bestimmter IMT-Medikamente (z. B. Tocilizumab) nicht zuverlässig.

Am häufigsten trifft dies hier zu:

- verminderte Lymphozytenzahl

- niedriges Albumin

- Abnahme des Hämoglobinspiegels

- Erhöhung des C-reaktiven Proteins (CRP)

- Erhöhung der Blutkörperchensenkungsgeschwindigkeit (BSG)
- Erhöhung der Laktatdehydrogenase (LDH)

- Erhöhung des D-Dimers

Bei schwerem COVID-19:

- verminderte Lymphozytenzahl

- vermindertes Albumin

- verringerte Hämoglobinspiegel

- Erhöhung des C-reaktiven Proteins (CRP)

- Erhöhung der Blutkörperchensenkungsgeschwindigkeit (BSG)

- Erhöhung der Laktatdehydrogenase (LDH)

- Erhöhung des D-Dimers

- Erhöhung der Neutrophilenzahl

- Erhöhung der Alaninaminotransferase (ALT)

- Erhöhung der Aspartataminotransferase (AST)

- Zunahme von kardialen Biomarkern (z. B. kardiale Troponine)

- Erhöhung von Procalcitonin

Interessenkonflikt

Die Autorinnen/Autoren geben an, dass kein Interessenkonflikt besteht.

Literatur

[1] Yang J, Zheng Y, Gou X et al. Prevalence of comorbidities in the novel Wuhan coronavirus (COVID-19) infection: a systematic review and meta-analysis. Int J Infect Dis 2020. doi:10.1016/j.ijid.2020.03.017

[2] Russell CD, Millar JE, Baillie JK. Clinical evidence does not support corticosteroid treatment for 2019-nCoV lung injury. Lancet 2020; 395: 473475

[3] Siddiqi HK, Mehra MR. COVID-19 illness in native and immunosuppressed states: a clinical-therapeutic staging proposal. J Heart Lung Transplant 2020; 39: 405-407

[4] Kampf G, Todt D, Pfaender S et al. Persistence of coronaviruses on inanimate surfaces and their inactivation with biocidal agents. J Hosp Infect 2020; 104: 246-251

[5] Li W, Moore M], Vasilieva N et al. Angiotensin-converting enzyme 2 is a functional receptor for the SARS coronavirus. Nature 2003; 426: 450 454

[6] Patel AB, Verma A. COVID-19 and angiotensin-converting enzyme inhibitors and angiotensin receptor blockers: what is the evidence? JAMA 2020. doi:10.1001/jama.2020.4812

[7] Ferguson NM, Laydon D, Nedjati-Gilani G; Imperial College COVID-19 Response Team. Impact of non-pharmaceutical interventions (NPIs) to reduce COVID-19 mortality and healthcare demand. London: Imperial College; 16 March 2020. Im Internet: https://www.imperial.ac.uk/ media/imperial-college/medicine/sph/ide/gida-fellowships/ImperialCollege-COVID19-NPI-modelling-16-03-2020.pdf; Stand: 16.07.2020

[8] D'Antiga L. Coronaviruses and immunosuppressed patients. The facts during the third epidemic. Liver Transpl 2020; 26: 832-834. doi:10.1002/It.25756

[9] Norsa L, Indriolo A, Sansotta $\mathrm{N}$ et al. Uneventful course in IBD patients during SARS-CoV-2 outbreak in northern Italy. Gastroenterology 2020. doi:10.1053/j.gastro.2020.03.062

[10] Bhoori S, Rossi RE, Citterio D et al. COVID-19 in long-term liver transplant patients: preliminary experience from an Italian transplant centre in Lombardy. Lancet Gastroenterol Hepatol 2020; 5: 532-533. doi:10.1016/S2468-1253(20)30116-3 
[11] Zhou Y, Fu B, Zheng $X$ et al. Pathogenic T cells and inflammatory monocytes incite inflammatory storm in severe COVID 19 patients. Natl Sci Rev 2020. doi:10.1093/nsr/nwaa041

[12] Mehta P, McAuley DF, Brown M et al. COVID-19: consider cytokine storm syndromes and immunosuppression. Lancet 2020; 395: 1033-1034. doi:10.1016/S0140-6736(20)30628-0

[13] Seah I, Agrawal R. Can the coronavirus disease 2019 (COVID-19) affect the eyes? A review of coronaviruses and ocular implications in humans and animals. Ocul Imm Infl 2020. doi:10.1080/09273948.2020. 1738501

[14] Wu P, Duan F, Luo $C$ et al. Characteristics of ocular findings of patients with coronavirus disease 2019 (COVID-19) in Hubei Province, China. JAMA Ophthalmol 2020; 138: 575-578. doi:10.1001/jamaophthalmol. 2020.1291

[15] Liu Z, Sun CB. Conjunctiva is not a preferred gateway of entry for SARS-CoV-2 to infect respiratory tract. J Med Virol 2020. doi:10.1002/ jmv.25859

[16] Sun CB, Wang YY, Liu GH et al. Role of the eye in transmitting human coronavirus: what we know and what we do not know. Front Public Health 2020; 8: 155. doi:10.3389/fpubh.2020.00155

[17] Navel V, Chiambaretta F, Dutheil F. Haemorrhagic conjunctivitis with pseudomembranous related to SARS-CoV-2. Am J Ophthalmol Case Rep 2020; 19: 100735. doi:10.1016/j.ajoc.2020.100735

[18] Marinho PM, Marcos AAA, Romano AC et al. Retinal findings in patients with COVID-19. Lancet 2020; 395: 1610. doi:10.1016/S0140-6736(20) 31014-X

[19] Casagrande M, Fitzek A, Püschel $K$ et al. Detection of SARS-CoV-2 in human retinal biopsies of deceased COVID-19 patients. Ocul Immunol Inflamm 2020. doi:10.1080/09273948.2020.1770301

[20] Lippi G, Plebani M. Laboratory abnormalities in patients with COVID2019 infection. Clin Chem Lab Med 2020; 58; 1131-1134. doi:10.1515/cclm-2020-0198
Weitere Quellen

[1] International Agency for the Prevention of Blindness. COVID-19 \& Eye Health. Im Internet: https://www.iapb.org/news/covid-19-resourceshere-is-what-we-know/; Stand: 16.07.2020

[2] American Academy of Ophthalmology. Important coronavirus updates for ophthalmologists. Im Internet: https://www.aao.org/headline/alertimportant-coronavirus-context; Stand: 16.07.2020

[3] American Academy of Ophthalmology. Coronavirus and Eye Care. Im Internet: https://www.aao.org/coronavirus; Stand: 16.07.2020

[4] World Health Organization. Rolling updates on coronavirus disease (COVID-19). Im Internet: https://www.who.int/emergencies/diseases/ novel-coronavirus-2019/events-as-they-happen; Stand: 16.07.2020

[5] Uveitis National Clinical Study Group. Uveitis Clinical Study Group COVID-19 Info Hub. Im Internet: https://www.uveitisstudygroup.org/? id=15; Stand: 16.07 .2020

[6] Casey Eye Institute. Best practice for prevention of droplet or contact transmission of infectious agents in ophthalmology clinics. Im Internet: https://www.ohsu.edu/sites/default/files/2020-03/CEl\%20Clinic\%20 Protocol\%20for\%20Suspect\%20or\%20Confirmed\%20COVID-19\%20 Patients\%202020-03-12.pdf; Stand: 16.07.2020

[7] University Health Network (UHN). UHN Droplet Precautions PPE Instructional Video. Im Internet: https://www.youtube.com/watch?v= Ww0Rf079MZ4; Stand: 16.07.2020

[8] MS Research Australia. An update from MS Research Australia on COVID-19. Im Internet: https://msra.org.au/news/ceo-message-covid19/; Stand: 16.07.2020 